\title{
Recent advances in all-optical signal processing in silicon photonics
}

\author{
Lawrence R. Chen ${ }^{1}$, Zifei Wang ${ }^{1}$, Junjia Wang ${ }^{1}$, Ivan Glesk ${ }^{2}$, and Rhys Adams ${ }^{3}$ \\ 1. Department of Electrical and Computer Engineering, McGill University, Montréal, QC H3A OE9 Canada \\ 2. Department of Electronic and Electrical Engineering, University of Strathclyde, Glasgow, Scotland, G1 1XU UK \\ 3. Department of Physics, CEGEP Vanier College, St. Laurent, QC H4L 3X9 Canada \\ lawrence.chen@mcgill.ca
}

\begin{abstract}
We review recent work on all-optical signal processing in silicon photonics. In particular, we describe the integration of a nonlinear optical loop mirror in silicon-on-insulator for applications including wavelength conversion, NRZ-to-RZ modulation format conversion, and optical time-division de-multiplexing.

OCIS codes: (070.4340) Nonlinear optical signal processing; (130.3120) Integrated optics devices
\end{abstract}

\section{Introduction}

The nonlinear optical loop mirror (NOLM) [1] has been studied for several decades and has many applications in optical communications and high speed all-optical signal processing [2], including, amongst others, wavelength conversion [3], pulse generation [4], and optical time division de-multiplexing [5]. Silicon has a number of desirable characteristics that make it suitable for nonlinear all-optical signal processing [6]. In order to satisfy the need for integrating all-optical signal processing subsystems, we realized an on-chip NOLM in silicon-on-insulator (SOI) and demonstrated its application for wavelength conversion of $10 \mathrm{~Gb} / \mathrm{s}$ return-to-zero on-off keying (RZ-

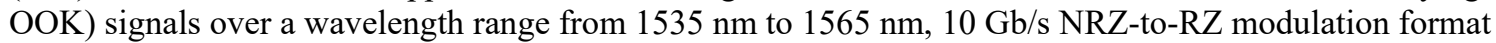
conversion, and optical time-division de-multiplexing of four $10 \mathrm{~Gb} / \mathrm{s}$ tributary channels from a $40 \mathrm{~Gb} / \mathrm{s}$ data RZOOK input signal.

\section{Device and experiments}

The structure we present in this paper is a cross-phase modulation (XPM)-based NOLM, whose principle is explained in [7]. A schematic of the on NOLM in SOI is shown in Fig. 1(b). The input and output are coupled using vertical grating couplers (VGCs) that are designed for C-band TE mode operation with a $3 \mathrm{~dB}$ bandwidth of $\sim 15 \mathrm{~nm}$ and a coupling loss of $7.5 \mathrm{~dB} / \mathrm{VGC}$. Ports 2 and 3 are connected to a multi-mode interferometer (MMI) coupler having a 50/50 coupling ratio of 50/50 and the length of nonlinear waveguide in the loop is $1.25 \mathrm{~cm}$. The nonlinear waveguide is based on a serpentine configuration with waveguide bends having a bend radius of $10 \mu \mathrm{m}$ and straight segments having a length of $2 \mathrm{~mm}$. Ports 1 and 4 are added to the beginning and end of the nonlinear waveguide via two 50/50 Y-branch power splitters and work as the input port for the pump pulses and a monitoring port for detection. The input signals are launched through Port 2 and Port 3 is used as the output. The device was fabricated using e-beam lithography with a single etch and the total size is $\sim 2.65 \mathrm{~mm} \times 0.41 \mathrm{~mm}$. The cross-section of the nonlinear and waveguide segments is shown in Fig. 1(a): the waveguides have a width of $500 \mathrm{~nm}$ and height of 220 $\mathrm{nm}$, which is designed to support largely the fundamental TE mode. All of the components sit on top of buried

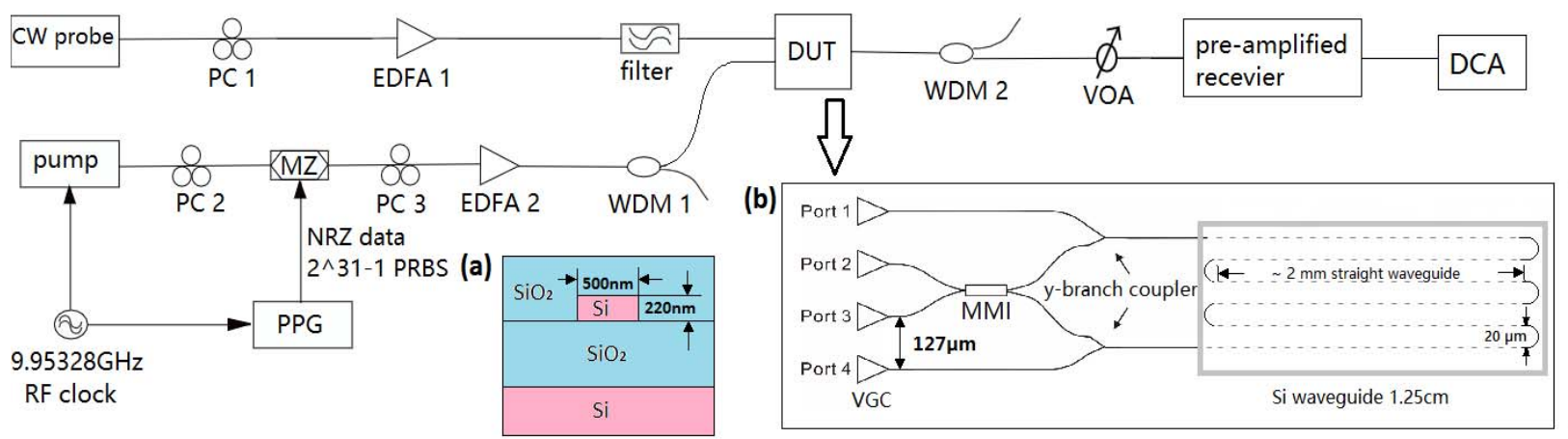

Fig.1. Schematic of the experimental setup for XPM-based wavelength conversion. Insets: (a) Cross-section of Si nanowire; (b) The structure of integrated NOLM device. PC: polarization controller; EDFA: erbium-doped fiber amplifier; DUT: device under test; VOA: variable optical attenuator; DCA: digial communications analyzer. 
oxide layer with a thickness of $3 \mu \mathrm{m}$ and are covered by a $2 \mu \mathrm{m}$ oxide cladding. The measured propagation loss of the waveguide is $\sim 2.7 \mathrm{~dB} / \mathrm{cm}$ and the total loss of the NOLM from Port 1 to Port 3 is $\sim 27 \mathrm{~dB}$.

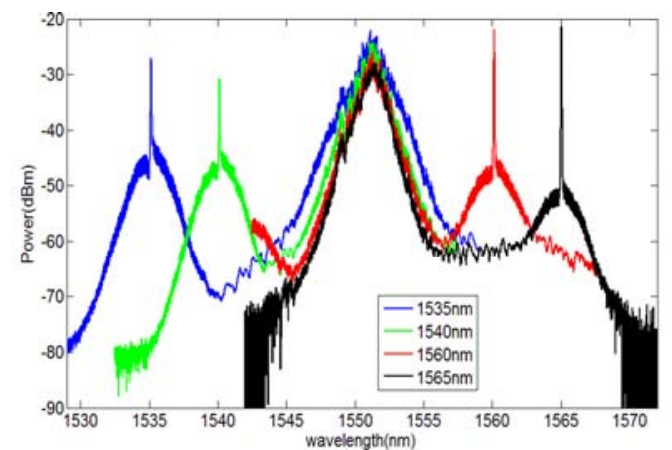

(a)

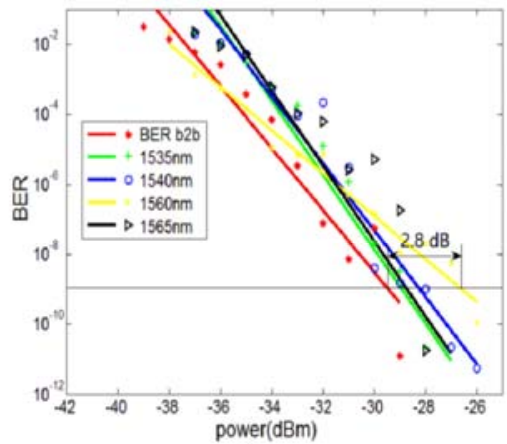

(b)

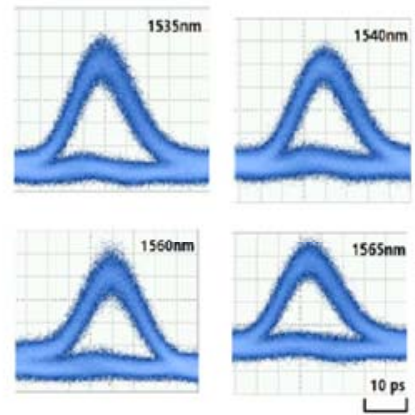

(c)

Fig. 2. (a) Spectra of the output of integrated NOLM. The pump is located at $\sim 1550 \mathrm{~nm}$ and the probe varies from $1535 \mathrm{~nm}$ to $1565 \mathrm{~nm}$. (b) Measured BER of the converted RZ-OOK signals and (c) eye diagrams measured at error-free operation.

The experimental setup for wavelength conversion is also illustrated in Fig 1. The pump is an RZ-OOK signal with a PRBS length of $2^{31}-1$ and is generated by a mode-locked laser at $9.95328 \mathrm{GHz}$ with a pulse width of 5 ps. The pulses are then modulated by a $\mathrm{LiNbO}_{3}$ Mach-Zehnder modulator (MZ) driven by a pulse pattern generator (PPG). The modulated pump is amplified by a high power EDFA and filtered by a WDM to remove the amplified spontaneous emission (ASE) noise. After amplifying and filtering, the pulses are broadened to 15 ps. The continuous wave $(\mathrm{CW})$ probe light from a tunable laser is also amplified, filtered, and coupled into the chip. The output signal from the NOLM is filtered by another WDM to remove the pump and the wavelength converted signals are detected using a pre-amplified receiver. We fix the central wavelength of the pump to $1550 \mathrm{~nm}$ and vary the probe wavelength from $1535 \mathrm{~nm}$ to $1565 \mathrm{~nm}$; bit error rate (BER) measurements are obtained for probe wavelengths of $1535 \mathrm{~nm}, 1540 \mathrm{~nm}, 1560 \mathrm{~nm}$, and $1565 \mathrm{~nm}$. The results are summarized in Fig. 2. We observed XPM-based broadening around the central wavelength of the probe as the probe wavelength is tuned over a $30 \mathrm{~nm}$ range. We obtained error-free operation and there is a clearly opened eye-diagram for the wavelength converted signals with power penalties less than $2.8 \mathrm{~dB}$.

Next, we demonstrate $10 \mathrm{~Gb} / \mathrm{s}$ NRZ-to-RZ modulation format conversion, see Fig. 3(a). The experimental setup is similar to that used for wavelength conversion except that a $10 \mathrm{~Gb} / \mathrm{s}$ NRZ-OOK signal is used as the input signal (rather than a CW signal) and the pump corresponds to the amplified mode-locked laser pulses. Figures 3(b) and (c) shows the BER and eye diagrams of original NRZ data and converted RZ signals. The results are similar to those obtained for wavelength conversion: there is a power penalty of $2.8 \mathrm{~dB}$ for the converted RZ signal.

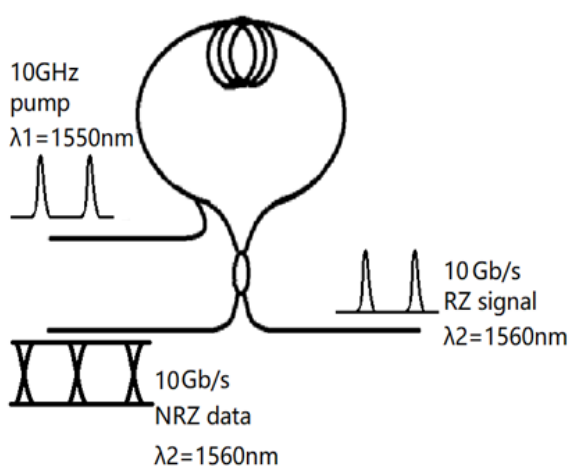

(a)

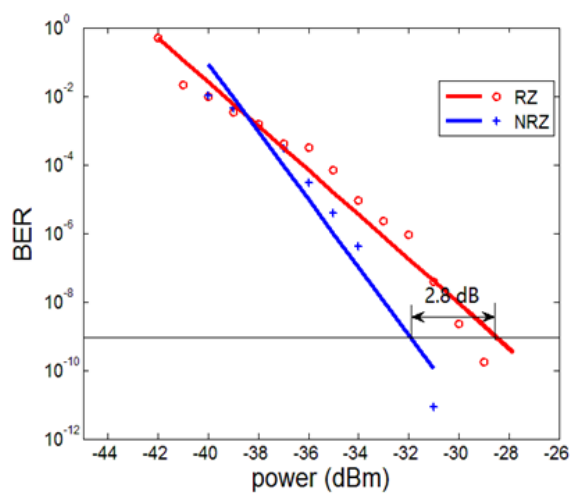

(b)

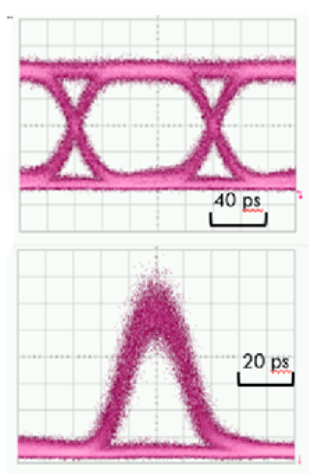

(c)

Fig. 3. (a) Principle of NRZ-to-RZ modulation format conversion, (b) measured BER vs. received power, and (c) eye diagrams at error-free operation of the original NRZ and converted RZ signals. 
Finally, we present optical time-division de-multiplexing of a $40 \mathrm{~Gb} / \mathrm{s}$ input signal to four $10 \mathrm{~Gb} / \mathrm{s}$ tributary channels. The principle is shown in Fig. 4(a). The input to the NOLM is $40 \mathrm{~Gb} / \mathrm{s}$ RZ-OOK data signal, which is generated by optically multiplexing a $10 \mathrm{~Gb} / \mathrm{s}$ RZ-OOK signal with a PRBS length of $2^{7}-1$; the pump is the same amplified pulse train as that used in the NRZ-to-RZ modulation format conversion experiment. BER measurements show that the four demultiplexed tributary channels exhibit no error floor and there is a maximum power penalty of $3.9 \mathrm{~dB}$. Clear open eye diagrams are obtained and error-free demultiplexing is achieved.

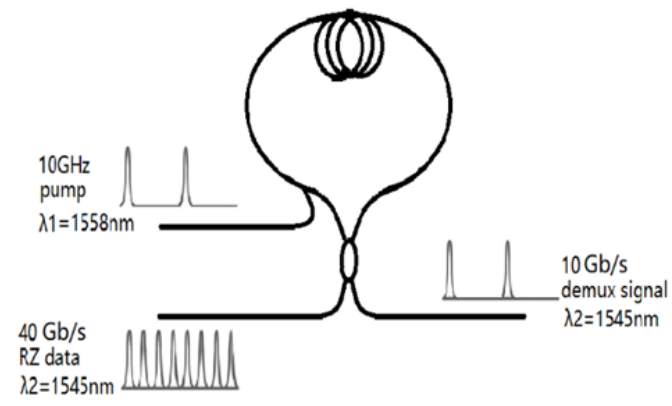

(a)

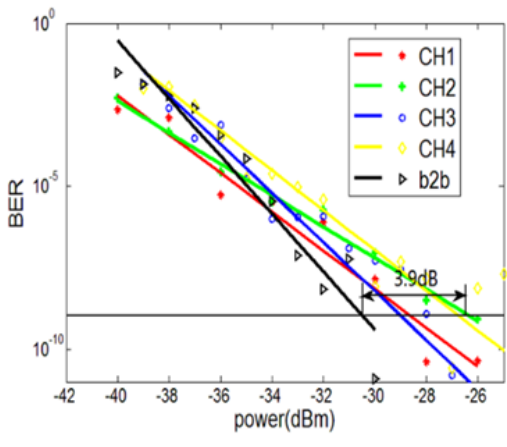

(b)

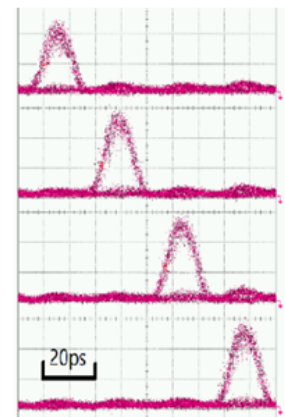

(c)

Fig. 4. (a) Principle of the reconfigurable optical time-division demultiplexing, (b) measured BER vs. received power for the four demultiplexed tributary channels and the back-to-back $40 \mathrm{~Gb} / \mathrm{s}$ data, and (c) eye diagrams of the four channels at error-free operation.

For the last two experiments, we fixed the pump and signal wavelengths. However, wavelength conversion over a $30 \mathrm{~nm}$ range was demonstrated and thus, we expect that NRZ-to-RZ modulation format conversion and optical time-division de-multiplexing can also operate over a broad wavelength range.

\section{Conclusion}

We have presented for the first time an on-chip NOLM in SOI and successfully demonstrated XPM-based wavelength conversion of $10 \mathrm{~Gb} / \mathrm{s}$ RZ-OOK signals, $10 \mathrm{~Gb} / \mathrm{s} \mathrm{NRZ-to-RZ} \mathrm{modulation} \mathrm{format} \mathrm{conversion,} \mathrm{and}$ optical-time-division de-multiplexing from $40 \mathrm{~Gb} / \mathrm{s}$ to $10 \mathrm{~Gb} / \mathrm{s}$. Error-free operation is obtained with a maximum power penalty of $3.9 \mathrm{~dB}$. The device is capable of supporting even higher rates of operation, e.g., optical time division demultiplexing at $160 \mathrm{~Gb} / \mathrm{s}$. We believe that XPM-based wavelength conversion in an on-chip NOLM will provide an advancement towards the integration of all-optical high-speed optical signal processing subsystems.

\section{Acknowledgement}

This project was support by the Natural Sciences and Engineering Research Council of Canada, the Fonds de Recherche du Québec - Nature et technologies, and the European Union's Horizon 2020 research and innovation program under the Marie Skłodowska-Curie grant agreement No. 734331.

\section{References}

[1] N. J. Doran and D. Wood, "Nonlinear optical loop mirror,” Opt. Lett. 13, 1, 56-58 (1988).

[2] S. Oda and A. Maruta, “All-optical digital-to-analog conversion using nonlinear optical loop mirrors,” IEEE Photon. Technol. Lett. 18.5, 703705 (2006).

[3] T. Sakamoto, F. Futami, K. Kikuchi, S. Takeda, Y. Sugaya and S. Watanabe, "All-optical wavelength conversion of 500-fs pulse trains by using a nonlinear-optical loop mirror composed of a highly nonlinear DSF," IEEE Photon. Technol. Lett. 13, 5, 502-504 (2001).

[4] T. Huang, J. Li, J. Sun and L. R. Chen, "Photonic generation of UWB pulses using a nonlinear optical loop mirror and its distribution over a fiber link," IEEE Photon. Technol. Lett. 23, 17, 1255-1257 (2011).

[5] H. Sotobayashi, C. Sawaguchi, Y. Koyamada and W. Chujo, "Ultrafast walk-off-free nonlinear optical loop mirror by a simplified configuration for 320-Gbit/s time-division multiplexing signal demultiplexing,” Opt. Lett. 27, 17, 1555-1557 (2002).

[6] J. Leuthold, C. Koos and W. Freude, “Nonlinear silicon photonics,” Nat. Photonics 4, 535 (2010).

[7] A. Bogoni, M. Scaffardi, P. Ghelfi and L. Poti, "Nonlinear optical loop mirrors: investigation solution and experimental validation for undesirable counterpropagating effects in all-optical signal processing," IEEE J. Sel. Topics in Quantum Electron. 10, 5, 1115-1123 (2004). 